Swarthmore College

Works

Physics \& Astronomy Faculty Works

Physics \& Astronomy

10-1-2006

\title{
Dipole Trapped Spheromak In A Prolate Flux Conserver
}

Michael R. Brown

Swarthmore College, doc@swarthmore.edu

C. D. Cothran

Jerome Fung , '06

M. Chang

Jason Alexander Horwitz , '07

See next page for additional authors

Follow this and additional works at: https://works.swarthmore.edu/fac-physics

Part of the Physics Commons

Let us know how access to these works benefits you

\section{Recommended Citation}

Michael R. Brown; C. D. Cothran; Jerome Fung , '06; M. Chang; Jason Alexander Horwitz , '07; M. J. Schaffer; J. Leuer; and E. V. Belova. (2006). "Dipole Trapped Spheromak In A Prolate Flux Conserver". Physics Of Plasmas. Volume 13, Issue 10. DOI: 10.1063/1.2356690

https://works.swarthmore.edu/fac-physics/107

This work is brought to you for free by Swarthmore College Libraries' Works. It has been accepted for inclusion in Physics \& Astronomy Faculty Works by an authorized administrator of Works. For more information, please contact myworks@swarthmore.edu. 
Authors

Michael R. Brown; C. D. Cothran; Jerome Fung , '06; M. Chang; Jason Alexander Horwitz, '07; M. J.

Schaffer; J. Leuer; and E. V. Belova 


\section{AIP | Physics of \\ Plasmas}

\section{Dipole trapped spheromak in a prolate flux conserver}

M. R. Brown, C. D. Cothran, J. Fung, M. Chang, J. Horwitz, M. J. Schaffer, J. Leuer, and E. V. Belova

Citation: Physics of Plasmas (1994-present) 13, 102503 (2006); doi: 10.1063/1.2356690

View online: http://dx.doi.org/10.1063/1.2356690

View Table of Contents: http://scitation.aip.org/content/aip/journal/pop/13/10?ver=pdfcov

Published by the AIP Publishing

\section{Articles you may be interested in}

Validation of single-fluid and two-fluid magnetohydrodynamic models of the helicity injected torus spheromak experiment with the NIMROD code

Phys. Plasmas 20, 082512 (2013); 10.1063/1.4817951

Stable spheromak formation by merging in an oblate flux conserver

Phys. Plasmas 17, 032510 (2010); 10.1063/1.3334324

Aspect-ratio effects in the driven, flux-core spheromak

Phys. Plasmas 16, 052506 (2009); 10.1063/1.3134064

Excitation of the centrifugally driven interchange instability in a plasma confined by a magnetic dipolea)

Phys. Plasmas 12, 055703 (2005); 10.1063/1.1888685

Effect of the vacuum vessel on magnetic measurements in TCABR tokamak

Rev. Sci. Instrum. 75, 5038 (2004); 10.1063/1.1807001

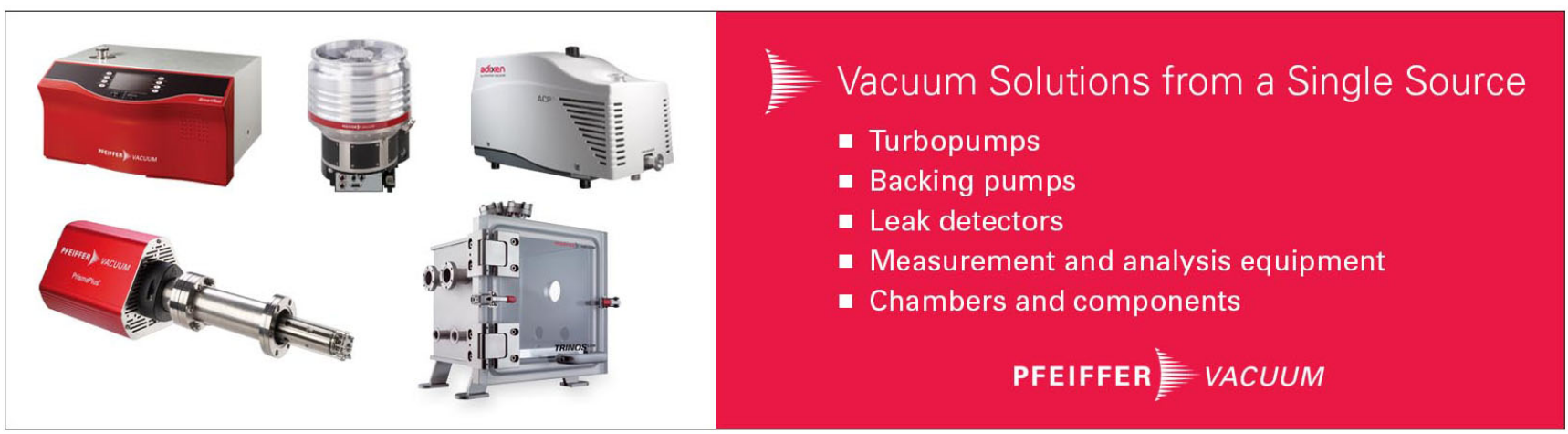




\title{
Dipole trapped spheromak in a prolate flux conserver
}

\author{
M. R. Brown, , ${ }^{\text {a) }}$ C. D. Cothran, J. Fung, M. Chang, and J. Horwitz \\ Department of Physics and Astronomy, Center for Magnetic Self Organization, Swarthmore College, \\ Swarthmore, Pennsylvania 19081
}

M. J. Schaffer and J. Leuer

General Atomics, P.O. Box 85608, San Diego, California 92186

E. V. Belova

Plasma Physics Laboratory, Princeton University, Princeton, New Jersey 08543

(Received 15 May 2006; accepted 28 August 2006; published online 12 October 2006)

This paper reports the observation and characterization of a spheromak formed in the Swarthmore Spheromak Experiment (SSX, [M. R. Brown, Phys. Plasmas 6, 1717 (1999)]) and trapped in a simple dipole magnetic field. The spheromak is studied in a prolate (tilt unstable) $0.4 \mathrm{~m}$ diameter, $0.6 \mathrm{~m}$ length copper flux conserver in SSX. This plasma does not tilt, despite the prolate flux conserver. The spheromak is characterized by a suite of magnetic probe arrays for magnetic structure $\mathbf{B}(\mathrm{r}, \mathrm{t})$, ion Doppler spectroscopy for $T_{i}$ and flow, and interferometry for $n_{e}$. Three-dimensional magnetohydrodynamics simulations of this configuration verify its gross stability. (C) 2006 American Institute of Physics. [DOI: 10.1063/1.2356690]

\section{INTRODUCTION}

Spheromaks ${ }^{1}$ are moderate $\beta$, compact, translatable, simply connected plasma configurations, all of which make them an attractive concept for a fusion reactor. When spheromaks are formed by a coaxial magnetized plasma gun, poloidal flux is amplified during the relaxation process. ${ }^{2}$ The spheromak is unstable to an ideal magnetohydrodynamic (MHD) tilt $^{3}{ }^{3}$ which must be stabilized in order to fulfill their potential for fusion. Spheromaks are typically stabilized by closefitting flux conservers. ${ }^{2-4}$ In order for a cylindrical flux conserver to be stabilizing, it must be oblate with length-toradius ratio $L / R \leqslant 1.67$. $^{5-7}$

The S-1 experiments at Princeton ${ }^{8}$ tried a variety of conducting boundaries to stabilize the tilt. These included figure-eight coils, unconnected solid disks as well as spoked "wagon wheels" above and below the spheromak, and finally full enclosure of the entire flux core by electrically connecting the two wagon wheels with conducting rods. ${ }^{9}$ None completely stabilized the tilt mode. It was found that line tying did reduce the growth of the tilt when a fraction of the spheromak closed flux intercepted the conducting boundary.

In early three-dimensional (3D) MHD simulation studies (motivated by the S-1 experiment), ${ }^{10}$ a variety of conducting boundaries were examined, including cylindrical walls and belts, metal disks, and central conductors. These simulations found complete stabilization of the tilt when enough closed flux of the spheromak was intercepted by the conducting wall of a cylindrical belt with radius smaller than the flux core. This could not, of course, be implemented experimentally as it would be incompatible with the inductive formation scheme. Stability is attributed to the energy required to bend line-tied field lines.

A close-fitting flux conserver was originally used in the

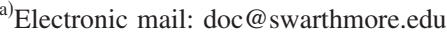

Los Alamos CTX spheromak. ${ }^{3}$ These early experiments met with limited success unless an oblate flux conserver was employed. ${ }^{1}$ In modern spheromaks (for example, $\operatorname{SSPX}^{4}$ ), a close-fitting, oblate $(L / R \cong 1)$ flux conserver is used. These spheromaks are ideally stable to the tilt (and all larger mode number instabilities) with no flux penetrating the conducting boundary. A notable result in this regard from the Beta-II spheromak $^{11}$ was the observation of a "flipped" state in an oblate flux conserver in a solenoidal field. The flipping occurred during formation in order to align the spheromak with the applied external field.

The Swarthmore Spheromak Experiment (SSX) has studied novel configurations in a prolate (tilt unstable) $0.4 \mathrm{~m}$ diameter, $0.6 \mathrm{~m}$ length, $3 \mathrm{~mm}$ wall copper flux conserver. ${ }^{12}$ Injection of a single spheromak into this flux conserver with a midplane vacuum dipole magnetic field generated with current in the same sense as the toroidal current in the spheromak generates an axisymmetric toroidal structure that is stable for the lifetime of the object, despite the elongated flux conserver. A three-dimensional MHD simulation verifies the stability of this object. Stabilization is likely due to the energetically favorable alignment of the toroidal current in the external field, line tying, and wall currents. These plasmas are characterized by $2 \times 10^{14} / \mathrm{cm}^{3}$ electron density, $T_{e}$, $T_{i} \approx 10-20 \mathrm{eV}$, and field strength $1 \mathrm{kG}$.

\section{EXPERIMENTAL AND NUMERICAL DETAILS}

\section{A. Experiment}

The SSX apparatus uses opposing, coaxial magnetized plasma guns at either end of a cylindrical copper flux conserver to produce spheromaks of either handedness (see Fig. 1). Only the east gun is active for the experiments described in this paper. Several different flux conservers have been used at SSX over the years with diameters varying between 0.17 and $0.5 \mathrm{~m}$. The present flux conserver diameter is 


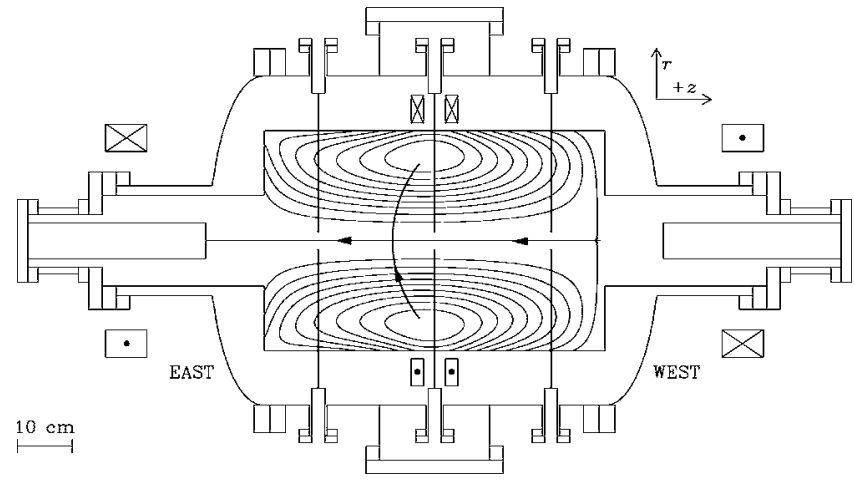

FIG. 1. Schematic of the Swarthmore Spheromak experiment showing the orientation of a single spheromak and six of the twelve magnetic probes (the other six are installed in the poloidal plane orthogonal to the one shown). The flux conserver is $0.4 \mathrm{~m}$ in diameter and $0.6 \mathrm{~m}$ in length. The dipole trapping coil (split) is at the midplane. The poloidal flux surfaces shown within the flux conserver are computed using the EQLFE Grad-Shafronov equilibrium solver.

$0.40 \mathrm{~m}$ with a length of $0.61 \mathrm{~m}$ (aspect ratio or elongation of 1.5 and $L / R=3$ ). The aspect ratio is deliberately chosen so that a spheromak should be tilt unstable $(L / R>1.67)$ for basic studies. Single spheromaks have previously been studied in SSX in oblate, tilt stable flux conservers. ${ }^{2}$ The guns are typically operated at $1.0 \mathrm{mWb}$ of magnetic flux, but during the plasma relaxation into a spheromak, poloidal flux is typically amplified to $\sim 3 \mathrm{mWb}$.

A pair of trapping coils at the midplane are used to produce the dipole vacuum field. The trapping coils have a mean diameter of $0.50 \mathrm{~m}$ and mean separation of $0.063 \mathrm{~m}$. A split pair, rather than a single coil, allows for diagnostic access. Each coil was housed in its own welded vacuum jacket. The on-axis field of these coils can be varied from 0 to $750 \mathrm{G}$ in both directions. The sign of the field used for our dipole trapping studies was such that the trapping coil current and the spheromak toroidal current were in the same sense: attractive. Oppositely directed image currents in the flux conserver walls complete the equilibrium.

Global magnetic structure of dipole-trapped spheromaks is studied with a set of 12 internal linear magnetic probes distributed as shown in Fig. 1. At each of three different axial locations, labeled east, midplane, and west, there are four linear probes inserted radially at $90^{\circ}$ intervals toroidally. Each probe consists of eight triplets of pickup coils sensitive to $B_{r}, B_{\theta}$, and $B_{z}$ at eight positions (at $2.54 \mathrm{~cm}$ increments) along the probe. The signals from these probes are actively integrated and acquired using a $1.25 \mathrm{MHz}$ multiplexing data acquisition system. ${ }^{13}$

Line averaged electron density is monitored with a quadrature HeNe laser interferometer. This diagnostic is sensitive to a diametrical chord through the plasma at the same east, midplane, or west axial locations as the magnetic probes.

Chord averaged ion flow and temperature $T_{i}$ are monitored with a fast ion Doppler spectroscopy (IDS) diagnostic. ${ }^{14}$ The combination of an echelle grating in the $1.33 \mathrm{~m}$ focal length spectrometer and a multi-anode photomultiplier tube (PMT) provide for the high resolution and fast time response necessary for these measurements. The IDS analyzes one view chord at the midplane per shot; ten view chords are available, ranging from almost diametrical to nearly tangential to the flux conserver (impact parameter $\approx 0$ to $20 \mathrm{~cm}$ ). The $C_{\mathrm{III}} 229.687 \mathrm{~nm}$ emission line is observed at 25 th order. The dispersion at the PMT for this setting is $0.0085 \mathrm{~nm} / \mathrm{mm}$ (each channel of the PMT is $1 \mathrm{~mm}$ wide). The bandwidth of the 12 instrumented channels is $\pm 65 \mathrm{~km} / \mathrm{s}$ and the instrument temperature is about $3.4 \mathrm{eV}$ (for $C$ ions). When not limited by signal strength, velocity resolution is better than $\pm 5 \mathrm{~km} / \mathrm{s}$. Signal strength (i.e., the brightness of this line) limits the time resolution to about $1 \mu \mathrm{s}$, which is faster than the characteristic MHD time scale $L / v_{A}$.

\section{B. Equilibrium calculations}

The equilibrium in Fig. 1 was calculated by solving the Grad-Shafranov equation in cylindrical coordinates using the finite-element code EQLFE. ${ }^{15}$ EQLFE was written especially to solve compact torus equilibria including at $r=0$. It is written in the Matlab software environment and uses the twodimensional partial differential equation capabilities available in Matlab. EQLFE is conventional in most other ways. The solution is found iteratively by alternating the plasma current and magnetic flux calculations. After each iteration, the total toroidal plasma current, $I_{p}$, is readjusted to the desired current. The computational domain is bounded by $r=0$ and elsewhere by an ideal magnetic flux-conserving boundary (the flux conserver, represented by straight line segments). Magnetic flux from specified current-carrying coils is calculated at the boundary before starting the iteration and kept as a boundary condition. The pressure gradient and poloidal current gradient functions are parametrized as powers of the poloidal flux.

\section{Three-dimensional MHD simulations}

Three-dimensional magnetohydrodynamic (MHD) simulations of dipole-trapped spheromaks have been performed for direct comparison with SSX data. In order to study the stability properties of this configuration, the 3D resistive MHD version of the HYM code has been used. ${ }^{16}$ The code solves MHD equations assuming uniform resistivity and viscosity profiles. The numerical values of the Lundquist number, $S=V_{A} R / \eta=1000$, and the Reynolds number, $R_{e}=V_{A} R / \nu=500$, are chosen to match the experimental parameters in SSX, where $V_{A}$ is the Alfvén velocity calculated using the edge magnetic field and the peak plasma density, and the length scale is the radius of the flux conserver $(R=0.2 \mathrm{~m})$. The simulations were performed using 128 grid points in radial and axial directions, and toroidal resolution up to $m=16$ toroidal mode numbers.

\section{RESULTS}

\section{A. Magnetics}

Figure 2 displays the global magnetic structure of an approximately axisymmetric spheromak trapped in an externally applied dipole field at the midplane. The dipole field 

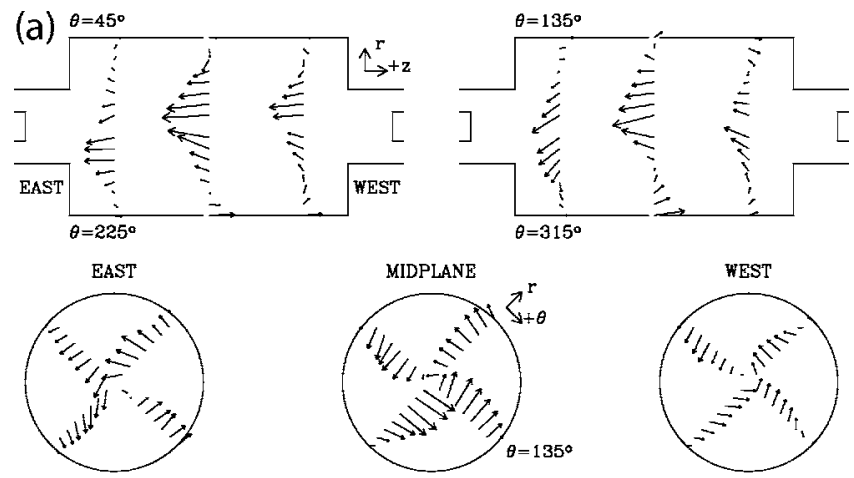

$\stackrel{1 \mathrm{kG}}{\longrightarrow}$

$\mathrm{t}=60.0 \mu \mathrm{s}$
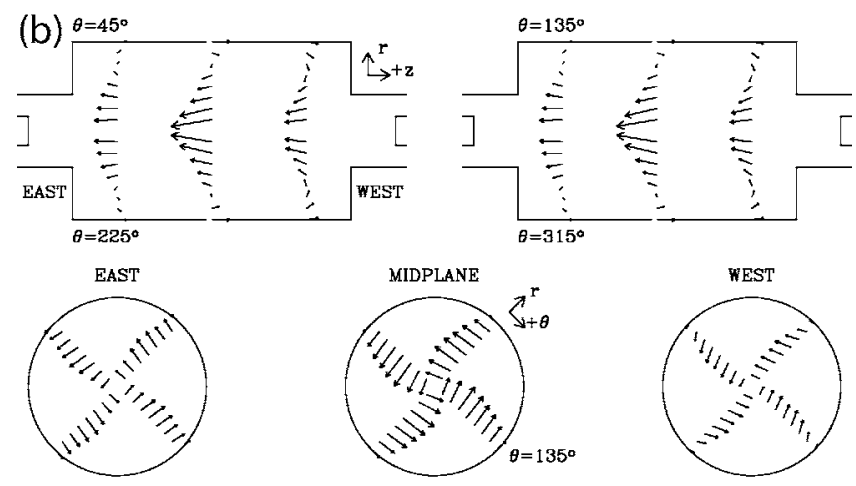

$\stackrel{1 \mathrm{kG}}{\longrightarrow}$

$\mathrm{t}=60.0 \mu \mathrm{s}$

FIG. 2. Global magnetic structure of the dipole trapped spheromak. Five views of the data are shown: two orthogonal $r z$ projections (top row), three $r \theta$ projections (bottom row) at $60 \mu \mathrm{s}$. For comparison, the full data (a) and the $m=0$ axisymmetric component (b) are both shown.

strength was about $150 \mathrm{G}$ field on axis (about $5 \mathrm{kA}$ turns of total current in the coils). Side and top views are depicted above, while the three axial views are depicted below. The time presented for this single spheromak shot is well into the relaxation and decay phase $(60 \mu \mathrm{s})$. Comparison of the full magnetic structure in Fig. 2(a) to the toroidally averaged $(m=0)$ component in Fig. 2(b) demonstrates the largely axisymmetric character of the configuration.

The structure remains axisymmetric throughout its lifetime, as demonstrated in Fig. 3. By $t \approx 30 \mu \mathrm{s}$, the average magnetic energy in the $m=0$ (solid lines) component exceeds the $m=1$ (dashed lines) component at the east, midplane, and west axial positions (red, green, and blue, respectively). Equilibrium appears to be established at $t \approx 50 \mu \mathrm{s}$. The structure seen in Fig. 2 is representative of the structure for $t \approx 50 \mu$ s through $t \approx 70 \mu \mathrm{s}$, when a change in the midplane $m=0$ magnetic energy is observed. This change appears as a decrease in the reversal of the axial field near the wall. The configuration continues to decay axisymmetrically for $t>70 \mu \mathrm{s}$. The energy decay time is approximately $15 \mu \mathrm{s}$.

In contrast, the magnetic structure observed for different dipole field strengths is dominantly nonaxisymmetric. The time dependence of the average magnetic energy in the $m$ $=1$ component to that in the $m=0$ component for the mid-

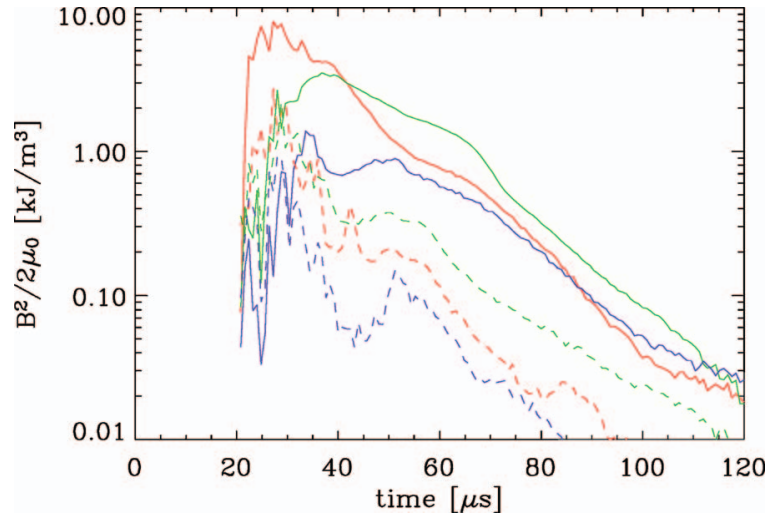

FIG. 3. (Color) The average energy in the $m=0$ component (solid lines) exceeds the average energy of the $m=1$ components (dashed lines) for the lifetime of the configuration. The east, midplane, and west are color coded red, green, and blue, respectively.

plane is shown in Fig. 4 for various dipole field strengths. The tilt mode is observed to grow rapidly for all other currents and polarities of the midplane coil.

\section{B. Flow and heating}

Figure 5(a) indicates a typical line shape from $C_{\mathrm{III}}$ emission of a dipole-trapped spheromak measured along a chord that should intercept all of the closed flux surfaces. The dashed line shows a Gaussian fit. The Doppler shift $(\Delta v)$ and width $\left(T_{i}\right)$ of the line shape obtained from the fits are shown as data points with error bars in Figs. 5(c) and 5(d). The moments of the line shapes are used to compute these quantities and are indicated as solid lines. The total line strength is shown in Fig. 5(b), as computed from the zeroth moment of the line shape. Chord-averaged flow speeds of approximately $10 \mathrm{~km} / \mathrm{s}$ are evident in every shot, and typical temperatures are between 10 and $30 \mathrm{eV}$ (see below).

The time dependence of the ion temperature averaged over ten shots for two different chords is shown in Fig. 6. One chord is the same as Fig. 5, while the other is along a

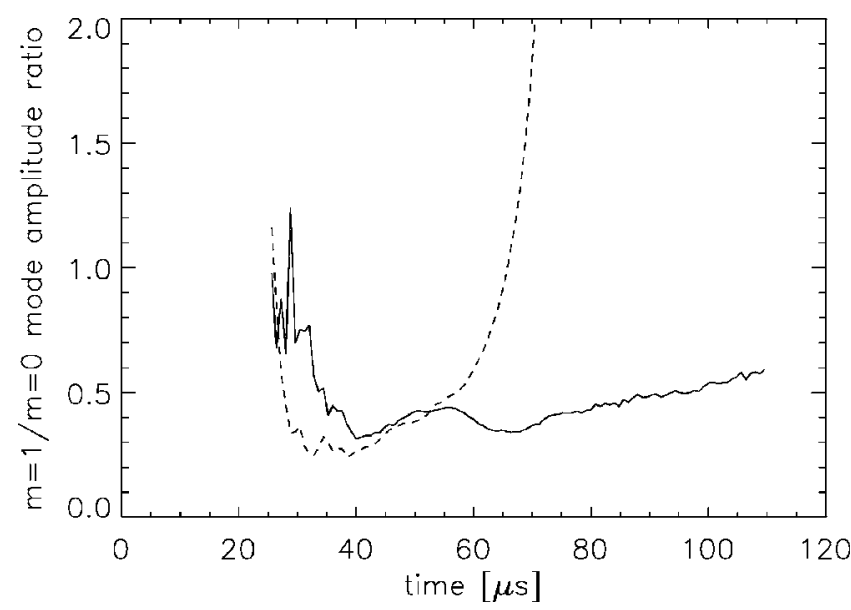

FIG. 4. Ratio of the $m=1$ to 0 mode energies for various currents in the midplane coils. A mostly axisymmetric configuration forms only for $5 \mathrm{kA}$ total current (solid); final states are dominantly $m=1$ for zero current (dashed) and for $10 \mathrm{kA}$ or more in the midplane coils (not shown). 

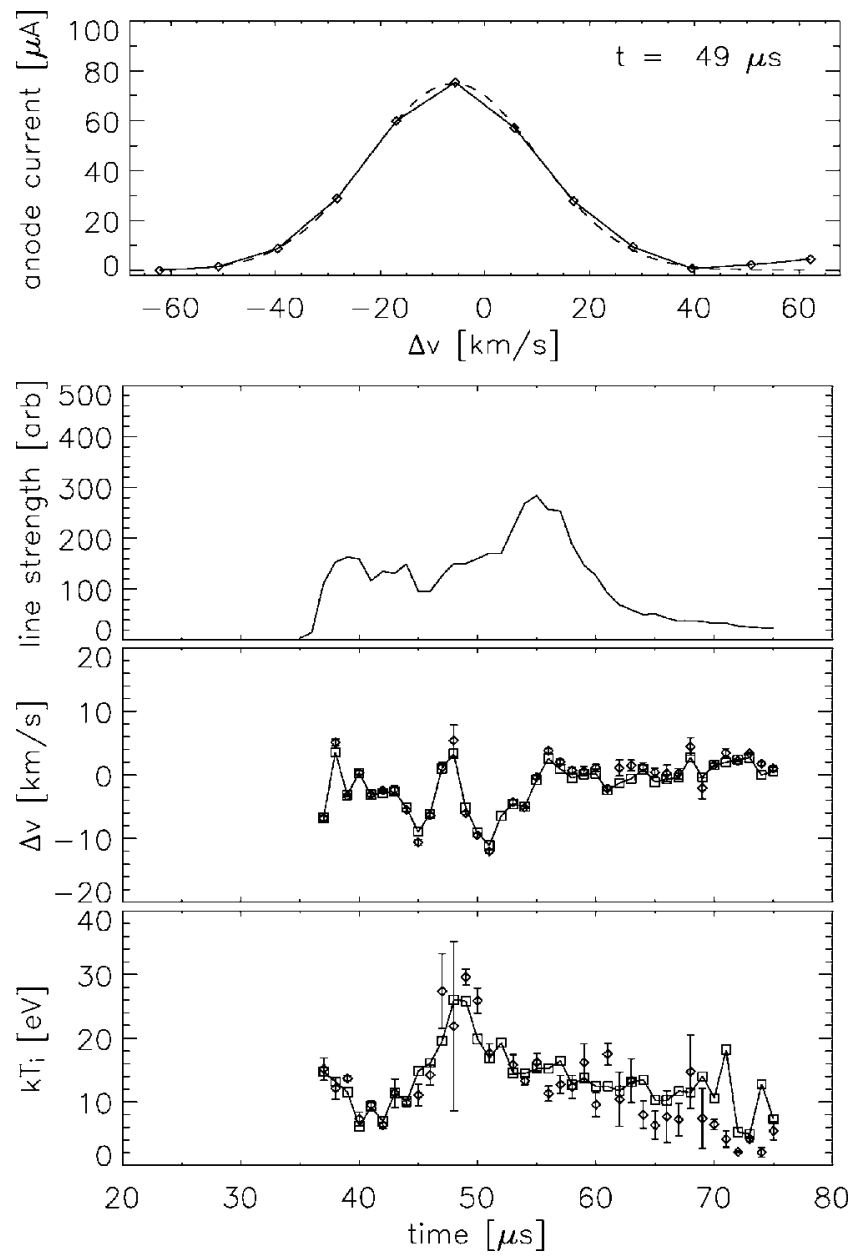

FIG. 5. Typical line shape (a) at $t=49 \mu$ s and the time dependence of the line intensity (b), line shift (c), and linewidth (d). The data points in (c) and (d) indicate the results of Gaussian fits to the line shapes, while the solid line indicates values computed from the first and second moments of the line shapes.

diameter (intercepting all flux surfaces). Both show the same time dependence; in fact, this is characteristic of the measurements for all chords. A clear epoch of systematic heating is evident as the spheromak forms during the interval from $t=35 \mu \mathrm{s}$ through $t=45 \mu \mathrm{s}$, followed by a slower decrease in ion temperature as the spheromak decays.

The uniform temperature profile is shown in Fig. 7(b) as well. The velocity profile (properly the dependence on chord impact parameter as no inversion has been performed on these data) in Fig. 7(a) also shows no features. These data have also been averaged over ten shots at each impact parameter, and averaged within a $5 \mu$ s window. Individual shots show much larger velocity fluctuations, as indicated in Fig. 5(c), than the averaged data of Fig. 7.

No Abel inversion is needed for the data of Figs. 7(a) and 7(b) given the flat profile in impact parameter. The line intensity, however, does show significant chord dependence. The data points in Fig. 8 show the measured line intensity (as a function of chord impact parameter), and the solid lines with shaded error bands indicate the radial dependence of the

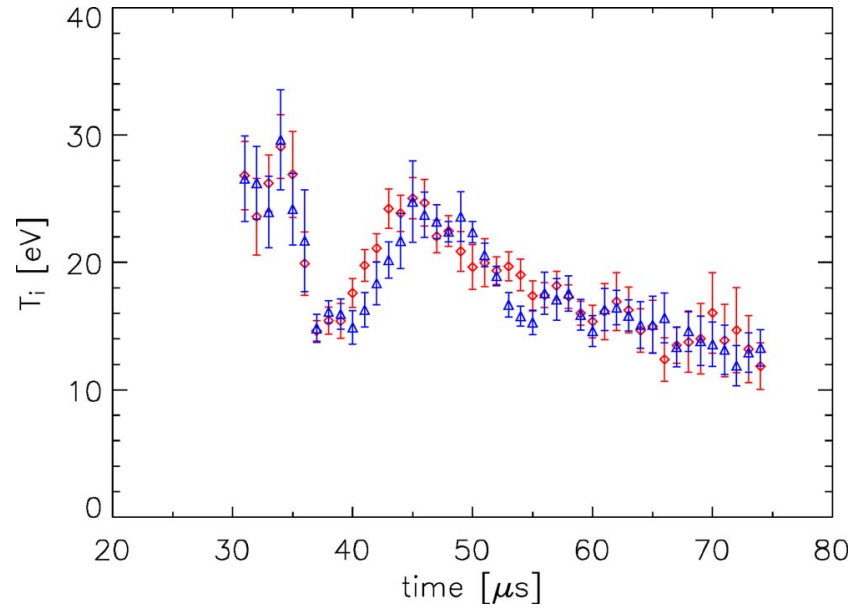

FIG. 6. Ion temperature averaged over ten externally identical shots for a view chord along a diameter (squares) and for a view chord with impact parameter at $8.4 \mathrm{~cm}$ (triangles). The latter view chord is insensitive to the expected open flux region and intercepts all of the expected closed flux region.

Abel inverted emissivity profile. The dashed lines show the chord integral of the emissivity profile, which are in agreement with the measured data.

The inversion procedure assumes the emissivity profile may be represented by a cubic spline with two intervals $r=0$ to $r=R / 2$ and $r=R / 2$ to $r=R$, where $R$ is the flux conserver radius (to avoid a cusp-like profile at $r=0$, the constraint that the profile have zero first derivative at $r=0$ is also required of the cubic polynomial in the first interval). This parametrization converts the Abel inversion problem into a linear least-squares-fitting problem that is straightforward to implement.

Figure 8(a) shows that early, just after formation, the profile is clearly peaked toward the plasma edge. Later, the emissivity profile is flatter with a small peak at $r=12 \mathrm{~cm}$, as indicated in Fig. 8(b). This peak is near the magnetic axis where electron density and temperature are also expected to

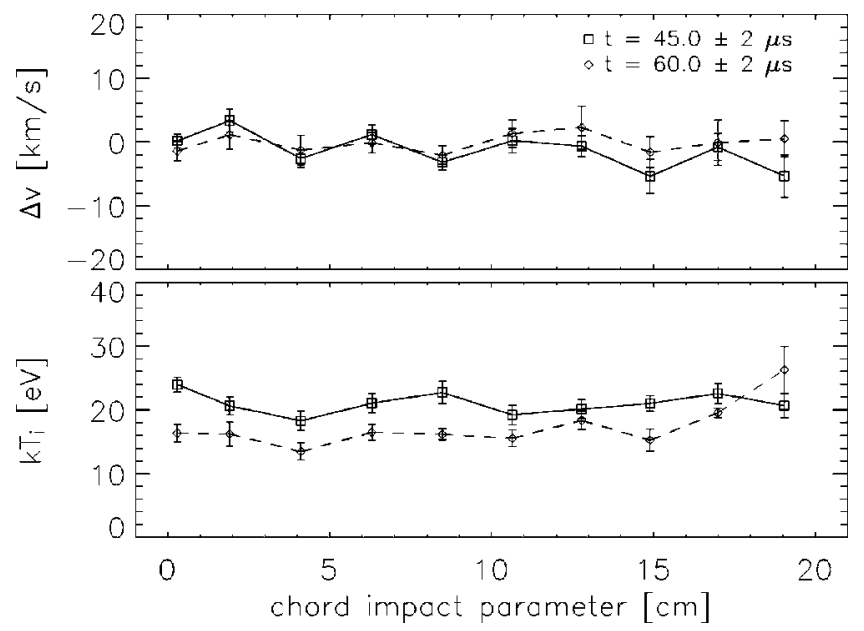

FIG. 7. Impact parameter dependence of flow (a) and ion temperature (b) at two times, $t=49 \mu \mathrm{s}$ when the ion temperature is greatest and $t=60 \mu \mathrm{s}$ during decay of the equilibrium. Data at each chord are averaged over ten externally identical shots and over a $5 \mu$ s window. 

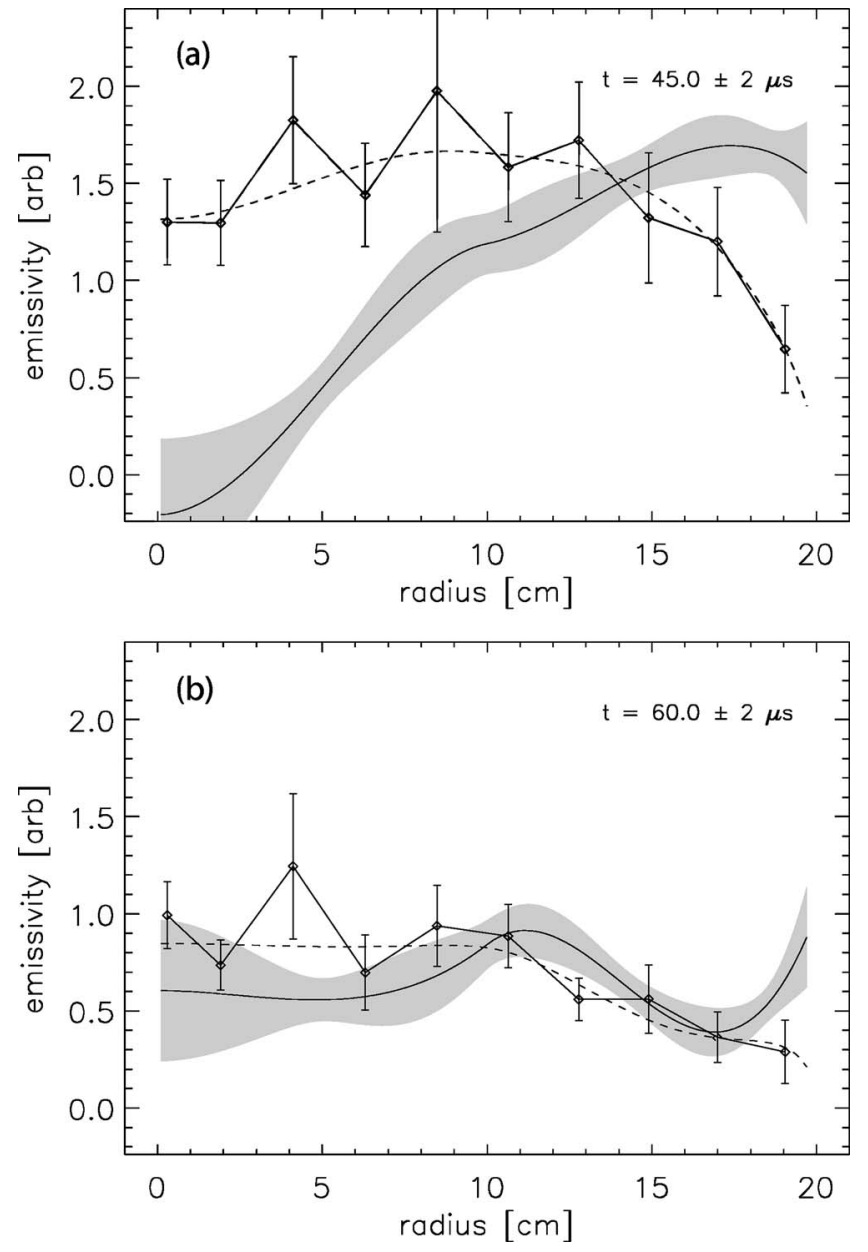

FIG. 8. Abel inverted emissivity profiles (solid line with shaded error band) at $t=49 \mu \mathrm{s}$ and at $t=60 \mu \mathrm{s}$. The measured dependence of line strength on impact parameter (data), as well as the Abel integral of the emissivity profile (dashed line), are overlayed for reference.

be peaked. The emissivity profile of Fig. 8 (b) persists at later times. These profiles indicate that the IDS measurements of temperature and flow are sensitive to local conditions along the line of sight through the plasma volume (rather than, for example, being limited only to emission from the edge).

\section{Equilibrium modeling}

For SSX dipole-trapped equilibrium calculations, the flux at the conserver came from the two midplane coils and a model of the fringing field of the two magnetized plasma guns. In the absence of plasma pressure profile data from the experiment, the computational plasma pressure profile was set arbitrarily as $p(\psi) \sim \psi^{1.2}$, where $\psi$ is the normalized poloidal flux ( $\psi=0$ at $r=0$ and the magnetic separatrix, and $\psi=1$ at the magnetic axis), but the pressure magnitude was adjusted by trial and error to approximately equal the measured plasma pressure. The poloidal current function derivative, $d\left(I^{2}\right) / d \psi$, was varied between $\psi^{1}$ and $\psi^{2}$, which yield broad and peaked toroidal current profiles, respectively. Note that $p=0$ and $I I^{\prime} \sim \psi^{1}$ yields the force-free Woltjer-Taylor relaxed equilibrium. The toroidal plasma current $I_{p}$ was adjusted by trial and error to match the measured axial magnetic field $B_{z}$ at $(r, z)=(0,0)$.
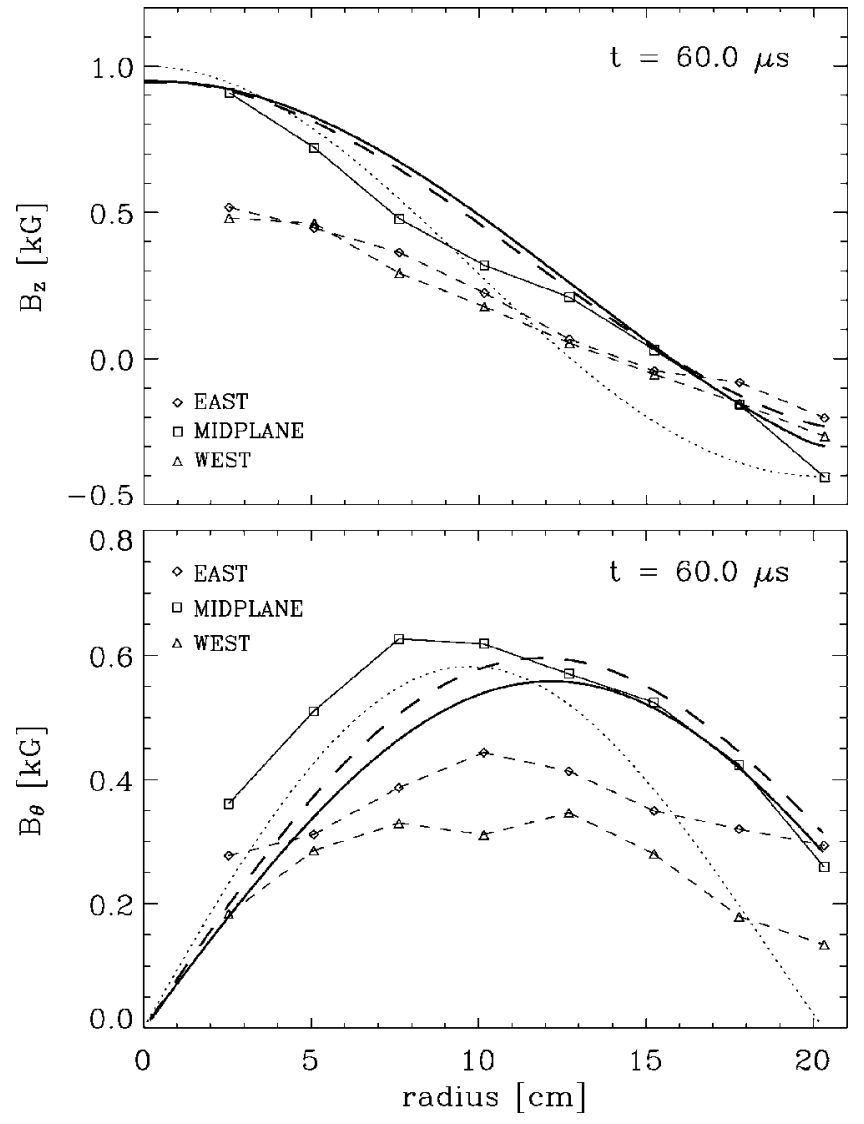

FIG. 9. Radial profiles of $B_{z}(r)$ (a) and $B_{\theta}(r)$ (b). Data are shown for the east, midplane, and west probe locations. The midplane equilibrium model calculations (green) for $\beta=0$ (long dashed) and $\beta=0.2$ (solid) are similar and agree with measurements at large $r$. For reference, the dotted lines indicate the Bessel function model for a spheromak in a cylindrical flux conserver with zero vacuum field.

The equilibrium of Fig. 1 was calculated with the broad current profile, $I I^{\prime} \sim \psi^{1}$. The total toroidal plasma current is $48.6 \mathrm{kA}$ and $\beta=0.2$, consistent with IDS $T_{i}$, interferometer $n_{e}$, and the magnetic structure measurements as described above. The comparison of the computed equilibrium and magnetic measurements is shown in detail in Fig. 9. The profiles of both $B_{z}$ and $B_{\theta}$ agree well with the equilibrium calculation at large radius, but differ at small radius. Little difference is found for the $\beta=0$ equilibrium corresponding to the Woltjer-Taylor state computed with the same external vacuum flux boundary condition (long dash). As a touchstone for intuition, the midplane profiles for the standard Bessel function model obtained for a cylindrical flux conserver with zero vacuum flux are displayed as well (dotted). Computed equilibria for peaked toroidal current profiles (not shown) disagree substantially with the data. Figure 10 shows the $q$ profile calculated from the broad profile equilibrium. Note that the $q$ profile dips slightly below unity in the outer part of the plasma, but the uncertainty of the equilibrium fit is such that it is not possible to say whether the actual $q$ was slightly less than or greater than unity.

The magnetic structure shown in Fig. 2 indicates the configuration is not symmetric axially. This is due to the fringing fields of the gun bias fields. Although the west gun was not active (the spheromak was formed from the east 


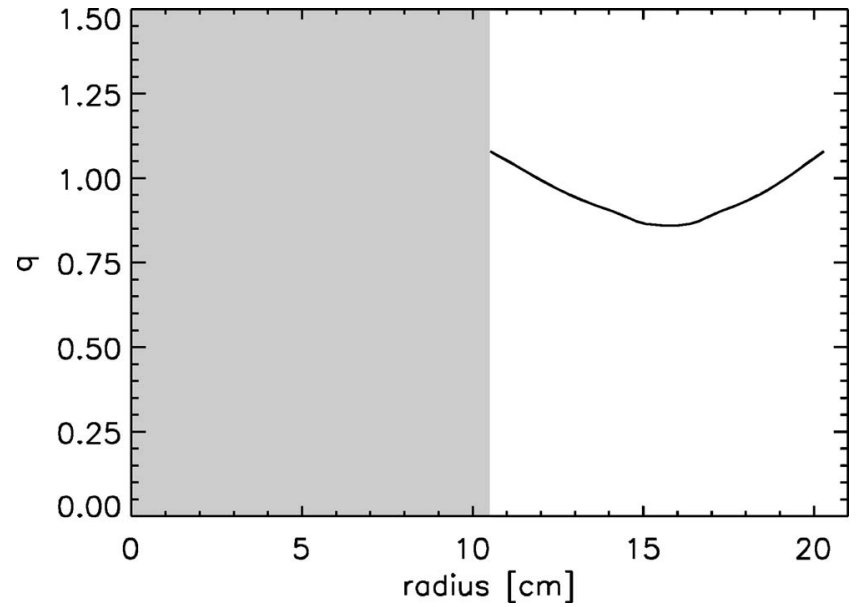

FIG. 10. Radial $q$ profile for the broad current profile equilibrium. The shaded region indicates where there are no closed flux surfaces.

gun), its bias field was applied. The weak vacuum field within the flux conserver volume generated by the two bias coils forms a cusp. Since the toroidal current of the spheromak was parallel to the current in the east bias coil (attractive) and antiparallel to the current in the west bias coil (repulsive), the equilibrium is not centered within the flux conserver. This also accounts for the oddly shaped flux surfaces for the equilibrium shown in Fig. 1.

\section{Stability calculation}

The axisymmetric dipole-trapped spheromak equilibrium described above with broad current profile has been used as an initial condition for the 3D nonlinear MHD simulations. Additional nonaxisymmetric random initial perturbation in plasma velocity has been added in order to allow excitation of MHD modes with different toroidal mode numbers $(m$ $=1-16)$ and various polarizations. The nonlinear evolution of initial configuration has been followed for about $t=25 \tau_{A}$, where $\tau_{A}=R / V_{A}$ is the Alfvén time. No growing MHD modes have been observed in the simulations. Time evolution plots (Fig. 11) of plasma kinetic energy for different Fourier harmonics show decay of the initial perturbation, and demonstrate the stability of a dipole-trapped spheromak configuration with respect to the interchange modes, the $m=1$ tilt mode, as well as $m>1$ co-interchange modes. The nonlinear numerical simulations, therefore, verify the experimentally observed gross stability of the configuration.

\section{DISCUSSION}

The object studied in this paper appears to be grossly in axisymmetric equilibrium, although it does contain an undesirably large nonaxisymmetric distortion. However, the relative amplitude of the $m=1$ component does not grow compared to the axisymmetric component. Stabilization of the tilt despite the prolate flux conserver is likely due to a combination of line-tying, favorable alignment in the applied vacuum field, and passive wall currents due to the nearby conducting wall.

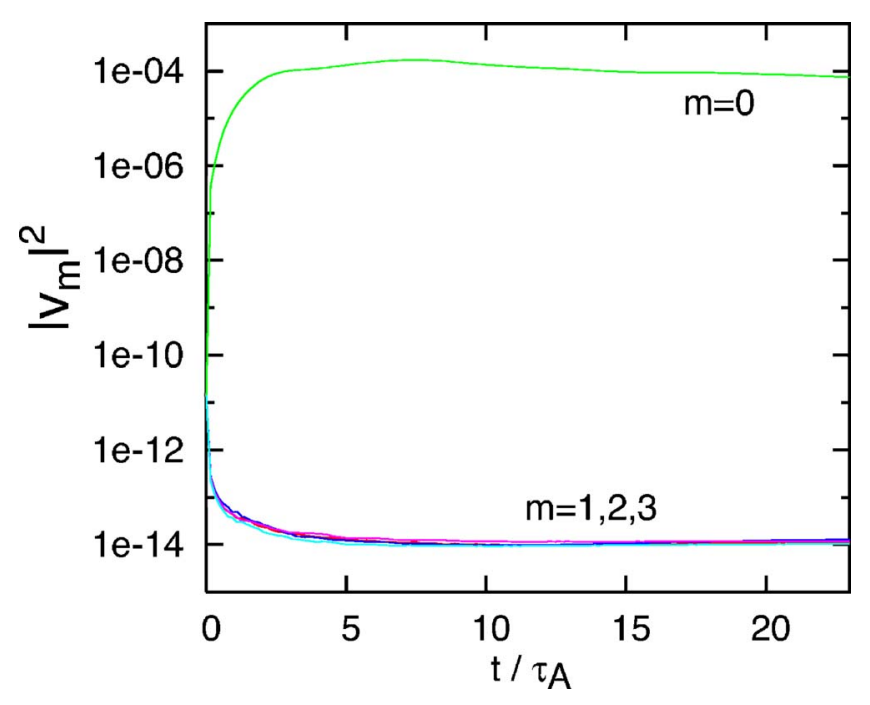

FIG. 11. Results of 3D MHD simulations. The time evolution of plasma kinetic energy for different Fourier harmonics show decay of the initial perturbation, and demonstrate the stability of the dipole-trapped spheromak configuration with respect to the interchange modes, the $m=1$ tilt mode, as well as $m>1$ co-interchange modes.

Comparison to the equilibrium calculation seems to suggest some open flux surfaces terminating at the cylindrical wall. The nonzero $B_{\theta}$ at the wall observed in the data of Figs. 2 and 9 clearly indicates the presence of current on poloidal flux surfaces that must close through the wall. These line-tied fields should be stabilizing in the same manner as was found for the case of the cylindrical belts in the simulations for the S-1 spheromak. ${ }^{10}$ No end walls were needed for stabilization in those studies; similarly, the end walls in this experiment are likely too remote due to the elongation to be contributing to the stabilization.

The hoop stress of the plasma toroidal current and pressure is balanced by axial field due to induced toroidal wall currents, but the applied vacuum external (dipole) field is in the opposite sense to the usual applied poloidal fields for magnetic confinement. The dipole field pulls the object toward the wall, and equilibrium is established when a sufficiently large wall current is induced. The magnetic axis is shifted to $r \approx 0.7 R$ compared to the Bessel function model at $r \approx 0.62 R$.

It appears that the applied vacuum field instead assists in stabilizing the tilt mode. In a simple analogy, the dipole moments formed by the toroidal current and the external dipole loop are stably aligned with respect to tilting in these new SSX experiments. From the point of view of external equilibrating fields, the external dipole current loop plus the induced toroidal current in the wall combine as a hexapole current distribution that apparently attracts the plasma into a magnetic well at the midplane. The total vertical field thus provides a restoring force, and the plasma is stable both to axisymmetric axial and to tilt displacements.

The bulk of the stabilization is likely due to a combination of the two mechanisms described above, line-tying and dipole-trapping. The reaction of nonaxisymmetric wall currents is certainly helpful, but their effectiveness is likely limited due to the split flux conserver. Reduction of the tilt 
growth rate in spheromaks due to rotation ${ }^{17}$ plays no role due to the absence of any dynamically relevant flow observed in the object described in this paper.

This object has some characteristics similar to a spheromak, but perhaps could be classified differently. Although the vacuum flux is about half that of the object, the object is largely produced by internal currents. The increase in $T_{i}$ during formation is characteristic of spheromaks and other selforganized plasmas. However, using the equilibrium calculation as a guide, the final equilibrium has a small closed flux volume compared to the volume of plasma on open flux surfaces. EQLFE indicates that $40 \%$ of the poloidal current is on open flux surfaces (about $16 \mathrm{kA}$ ).

Since most of the poloidal and toroidal current is outside the last closed flux surface, this configuration is more like a small stabilized pinch enclosing a large plasma flux core. In fact, through the closed flux region, the toroidal field is several times larger than the poloidal field. $r B_{T}$ is also roughly constant through this region. The closed flux region therefore resembles a pinch or tokamak. Note that it may be possible to exploit this feature and drive poloidal current by biasing the two halves of the flux conserver, thus converting this object into an ultrasmall aspect ratio spherical torus with a plasma center conductor, as suggested recently by Hsu. ${ }^{18}$

\section{SUMMARY}

Stable, prolate axisymmetric spheromaks have been formed and stably trapped in the combination of a simple, static dipole magnetic field and conducting wall image currents at SSX. The Grad-Shafranov equilibria are calculated to approximately fit the magnetic and pressure data and thus delineate the possible size and shape of the spheromak. The spheromak remains stable for the lifetime of the discharge despite the large elongation of the flux conserver $(L / R=3$ $>1.67)$. Azimuthal flow velocities measured with ion Doppler spectroscopy are relatively low $(10 \mathrm{~km} / \mathrm{s})$ and ion tem- perature peaks at about $T_{i}=25 \mathrm{eV}$. The magnetic field at the geometric axis is about $0.1 \mathrm{~T}$ and the density is about $2 \times 10^{14} \mathrm{~cm}^{-3}$, so $\beta \cong 0.2$. A fully three-dimensional MHD simulation has verified the gross stability of the configuration. Stability is likely due to a combination of line-tying, trapping in the applied dipole vacuum field, and passive wall currents.

\section{ACKNOWLEDGMENTS}

This work was supported by grants from the Department of Energy, the National Science Foundation, and General Atomics IR\&D. The authors gratefully acknowledge the assistance of V. Swisher and D. Cohen.

${ }^{1}$ T. R. Jarboe, Plasma Phys. Controlled Fusion 36, 945 (1994).

${ }^{2}$ C. G. R. Geddes, T. W. Kornack, and M. R. Brown, Phys. Plasmas 5, 1027 (1998).

${ }^{3}$ T. R. Jarboe, I. Henins, H. W. Hoida, R. K. Linford, J. Marshall, D. A. Platts, and A. R. Sherwood, Phys. Rev. Lett. 45, 1264 (1980).

${ }^{4}$ H. S. McLean, S. Woodruff, E. B. Hooper et al., Phys. Rev. Lett. 88, 125004 (2002)

${ }^{5}$ P. M. Bellan, Spheromaks (Imperial College Press, London, 2000).

${ }^{6}$ A. Bondeson, G. Marklin, Z. G. An, H. H. Chen, Y. C. Lee, and C. S. Liu, Phys. Fluids 24, 1682 (1981).

${ }^{7}$ J. M. Finn, W. M. Manheimer, and E. Ott, Phys. Fluids 24, 1336 (1981).

${ }^{8}$ M. Yamada, H. P. Furth, W. Hsu et al., Phys. Rev. Lett. 46, 188 (1981).

${ }^{9}$ F. J. Wysocki, Phys. Fluids 30, 482 (1987).

${ }^{10}$ T. Hayashi and T. Sato, Phys. Fluids 28, 3654 (1985).

${ }^{11}$ W. C. Turner, G. C. Goldenbaum, E. H. A. Granneman, J. H. Hammer, C. W. Hartman, D. S. Prono, and J. Taska, Phys. Fluids 26, 1965 (1983).

${ }^{12}$ C. D. Cothran, A. Falk, A. Fefferman, M. Landreman, M. R. Brown, and M. J. Schaffer, Phys. Plasmas 10, 1748 (2003).

${ }^{13}$ M. Landreman, C. D. Cothran, M. R. Brown, M. Kostora, and J. T. Slough, Rev. Sci. Instrum. 74, 2361 (2003).

${ }^{14}$ C. D. Cothran, J. Fung, M. R. Brown, and M. J. Schaffer, Rev. Sci. Instrum. 77, 063504 (2006).

${ }^{15}$ J. A. Leuer, M. J. Schaffer, P. B. Parks, and M. R. Brown, Bull. Am. Phys. Soc. 47, 275 (2002).

${ }^{16}$ E. V. Belova, S. C. Jardin, H. Ji, M. Yamada, and R. M. Kulsrud, Phys. Plasmas 7, 4996 (2000).

${ }^{17}$ U. Shumlak, T. K. Fowler, and E. C. Morse, Phys. Plasmas 1, 643 (1994).

${ }^{18} \mathrm{~S}$. Hsu and X. Z. Tang, in Innovative Confinement Concepts Workshop (2006). 